\title{
SCIENTIFIC REPORTS

\section{OPEN Epidemiology and Diversity of Rickettsiales Bacteria in Humans and Animals in Jiangsu and Jiangxi provinces, China}

Received: 21 February 2019

Accepted: 19 August 2019

Published online: 11 September 2019

\author{
Miao $\mathrm{Lu}^{1}$, Feng $\mathrm{Li}^{2}$, Yong Liao ${ }^{3}$, Jin-Jin Shen ${ }^{2}$, Jian-Min $\mathrm{Xu}^{4}$, Yin-Zhong Chen ${ }^{2}$, Jian-Hua $\mathrm{Li}^{3}$, \\ Edward C. Holmes $\mathbb{1}^{1,5,6}$ \& Yong-Zhen Zhang ${ }^{1,6}$
}

Diseases caused by Rickettsiales bacteria are a global public health problem. To better understand the diversity and origins of Rickettsiales infection in humans and animals, we sampled 134 febrile patients, 173 rodents and 43 shrews, as well as 358 ticks, from two cities in Jiangsu and Jiangxi provinces, China. Our data revealed a relatively high prevalence of scrub typhus cases in both localities. In addition, both serological tests and genetic analysis identified three patients infected with Anaplasma bovis, Rickettsia monacensis, and Orientia tsutsugamushi bacteria. Molecular epidemiological investigation revealed the co-circulation of multiple species of Rickettsiales bacteria in small mammals and ticks in both provinces, potentially including novel bacterial species. In sum, these data demonstrate the ongoing importance of Rickettsiales infection in China and highlight the need for the regular surveillance of local arthropods, mammals and humans.

Rickettsiales bacteria are obligate intracellular parasites of eukaryotes and responsible for a wide range of important human diseases including anaplasmosis, ehrlichiosis, rickettsioses, and scrub typhus ${ }^{1}$. Importantly, rickettsial diseases have not been effectively controlled worldwide, and scrub typhus remains a major public health concern in most countries where it is endemic ${ }^{2}$. In both developed and developing countries the incidence of human monocytotropic ehrlichiosis (HME) and human granulocytic anaplasmosis (HGA) have increased steadily since their discovery in the 1980s and 1990s, respectively ${ }^{3-6}$. In addition, the application of molecular diagnostic methods in recent decades has resulted in a continual increase in the identification of novel rickettsial bacteria from various samples, as well as their associated diseases ${ }^{2,7-9}$, and bacteria previously considered non-pathogenic are now commonly associated with human diseases ${ }^{2,8-10}$.

In China, the Rickettsiales Orientia tsutsugamushi, Rickettsia prowazekii and R. typhi have been important causes of past human morbidity and mortality $2,11,12$ and at least 5,000 human cases (range 5,041 23,540) were recorded each year over the last decade. Of these, scrub typhus is particularly important and has a wide geographic distribution ${ }^{13}$. Although the disease was originally considered to mainly occur in southern China ${ }^{14}$, cases of scrub typhus are now often reported in the northern China ${ }^{15,16}$, reflecting the northward spread of the disease. Human cases of HGA and HME have also been frequently reported since their discovery in Anhui province (in 2006) and Inner Mongolia (in 1999), respectively ${ }^{17,18}$. However, the true number of human infections caused by these rickettsial agents is uncertain due to a lack of commercial diagnostic kits and limited surveillance at both local and national levels. Finally, multiple species of Rickettsiales bacteria associated with human disease have

\footnotetext{
${ }^{1}$ State Key Laboratory for Infectious Disease Prevention and Control, Collaborative Innovation Center for Diagnosis and Treatment of Infectious Diseases, National Institute for Communicable Disease Control and Prevention, Chinese Center for Disease Control and Prevention, Changping, Beijing, China. ${ }^{2}$ Yancheng Center for Disease Control and Prevention, Yancheng, Jiangsu province, China. ${ }^{3}$ Ganzhou Center for Disease Control and Prevention, Ganzhou, Jiangxi province, China. ${ }^{4}$ Jiangxi Center for Disease Control and Prevention, Nanchang, Jiangxi province, China. ${ }^{5}$ Marie Bashir Institute for Infectious Diseases and Biosecurity, Charles Perkins Centre, School of Life and Environmental Sciences and Sydney Medical School, The University of Sydney, Sydney, NSW, 2006, Australia. ${ }^{6}$ Shanghai Public Health Clinical Center \& Institutes of Biomedical Sciences, Fudan University, Shanghai, China. Miao Lu, Feng Li and Yong Liao contributed equally. Correspondence and requests for materials should be addressed to Y.-Z.Z. (email: zhangyongzhen@icdc.cn)
} 


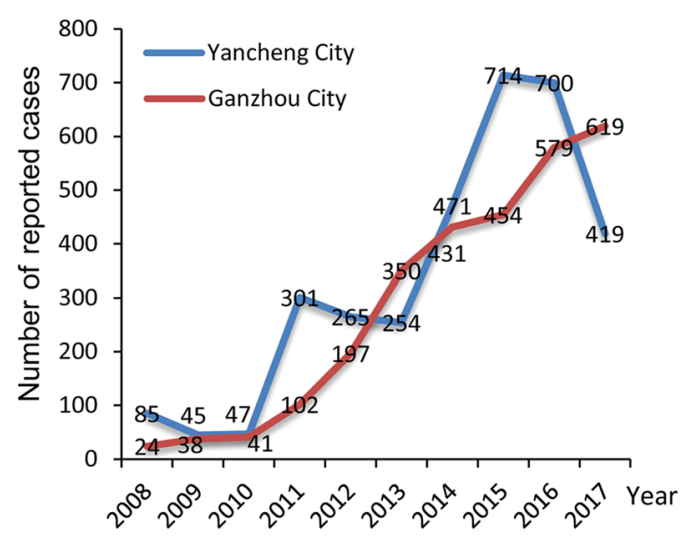

Figure 1. Annual number of cases of scrub typhus in Yancheng and Ganzhou cities, China, 2008-2017.

recently been identified throughout $\mathrm{China}^{12}$, again emphasizing that rickettsial disease is a major public health problem in this country.

Ticks and rodents play important roles in the transmission of Rickettsiales bacteria in animals and from ticks to humans $s^{19,20}$, and recent surveys have revealed a remarkable diversity, prevalence, and geographic distribution of Rickettsiales bacteria in both ticks and rodents ${ }^{2,11,12,20}$. China is home to a great diversity of ticks, with those identified to date being classified into at least 117 species (belonging to 10 genera of 2 families) and with a nationwide distribution ${ }^{21,22}$. The diversity of rodent species in China is also striking, with 171 recorded species ${ }^{23}$.

To better understand the diversity and origins of rickettsial infection in humans in China, particularly their relationship to those co-circulating bacteria present in small mammals and ticks, we collected blood from patients presenting with fever, and concurrently sampled ticks, rodents and shrews from Ganzhou city (Jiangxi province) and Yancheng city (Jiangsu province). Our results revealed that human cases were due to infection by Rickettsia monacensis, Anaplasma bovis, and O. tsutsugamushi, and that the local ecology of both regions was characterized by the co-circulation of a diverse range of Rickettsiales bacteria in small mammals.

\section{Results}

Occurrence of rickettsial disease in Jiangsu and Jiangxi provinces, China. Rickettsiales diseases have been reported in both Jiangsu and Jiangxi provinces since the 1980s. At least two major epidemics of scrub typhus have occurred in Yancheng city, Jiangsu province. The first epidemics occurred in 1986, with 264 registered clinically diagnosed cases. Fortunately, the number of cases then declined and was relatively stable for the subsequent 20 years (1986-2005). However, the true number of scrub typhus cases is likely higher than recorded because of a combination of inadequate testing and suboptimal reporting. A second epidemic of scrub typhus occurred in 2006, with 149 reported cases. Since then, some 3301 scrub typhus cases have been recorded over the last decade in Yancheng city (Fig. 1), reflecting the high prevalence of O. tsutsugamushi in animals in Yancheng. In the case of Ganzhou city (Jiangxi province), a total of 2835 clinically diagnosed scrub typhus cases were recorded between 2008-2017, with an annual increase in the number of cases since 2010 (Fig. 1). Due to a lack of commercial diagnostic kits, clinical cases caused by other Rickettsiales bacteria were not recorded.

Clinical features of Rickettsiales infection. Between 2013-2015, 72 and 62 blood samples were collected from patients with suspected Rickettsiales infection from two hospitals in Ganzhou and Yancheng cities, respectively (Fig. 2). Of these, $61.19 \%(82 / 134)$ were male and $38.81 \%(56 / 134)$ were female, with an age range from 12-88 years. All the patients had fever, and some had a series of other symptoms, including headache (80\%), dizziness (74\%), myalgia (85\%), rash (72\%), eschar (70\%), and lymphadenopathy (65\%).

Rickettsiales bacteria identified in patients. Blood samples were collected at day 1 of admission (3-8 days post the onset of fever). Specific IgG and IgM antibodies against spotted fever group Rickettsiae (SFGR), Anaplasma phagocytophilum, O. tsutsugamushi, Ehrlichia chaffeensis, R. prowazekii, and R. typhi in sera were detected for each of 134 patients by IFA assays. As described in Table 1, two patients from Jiangsu were strongly positive for SFGR and O. tsutsugamushi, respectively, while one patient from Jiangxi was positive for A. phagocytophilum. However, the levels of specific IgG and IgM antibody against these three agents were relatively lower in remaining patients than three positive patients. Finally, antibody tests against E. chaffeensis, R. prowazekii, and R. typh $i$ were all negative. Combined, these data suggest that the three patients might be infected with SFGR, $O$. tsutsugamushi and A. phagocytophilum.

To better determine the etiologic agents in these 134 patients, DNA extracted from their blood were screened for both the 16S ribosomal RNA (rrs) and $56 \mathrm{kDa}$ type-specific antigen (TSA56) genes. Consequently, the rrs gene was recovered from two patients - JSHMFN-77 and JXHFRJ-71 - while the TSA56 genes was obtained from the patient JSHFFN-74. However, our attempts to amplify either the rrs or the TSA56 gene from the remaining patients failed. Genetic analysis (blastn with Nucleotide collection nr/nt) of the recovered $r r s$ and TSA56 gene sequences revealed that they were most closely related to those of $R$. monacensis (SFGR) (99.41\%), A. bovis (98.75\%), and O. tsutsugamushi (99.44\%), respectively (see Supplementary Table S1). Hence, these data indicate that three patients were infected with R. monacensis, A. bovis, and O. tsutsugamushi. In addition, these three 


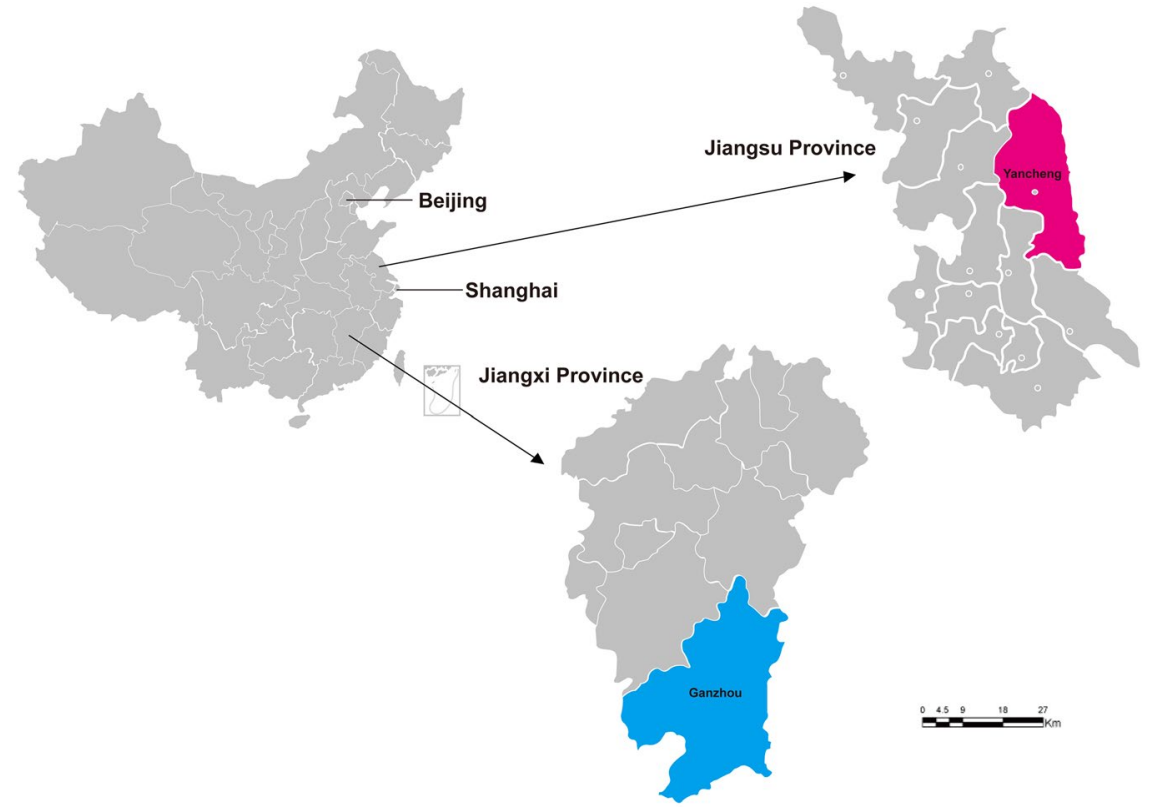

Figure 2. Map showing the location of sample collection sites in Yancheng city (Jiangsu province) and Ganzhou city (Jiangxi province), China.

\begin{tabular}{|c|c|c|c|c|c|c|}
\hline \multirow{2}{*}{\multicolumn{2}{|c|}{ Pathogen }} & \multicolumn{3}{|l|}{ Jiangsu } & \multicolumn{2}{|l|}{ Jiangxi } \\
\hline & & \multirow{2}{*}{\begin{tabular}{|l|}
$\begin{array}{l}\text { Patient } \\
\text { JSHMFN-77 }\end{array}$ \\
$1: 2048$ \\
\end{tabular}} & \multirow{2}{*}{$\begin{array}{l}\begin{array}{l}\text { Patient } \\
\text { JSHFFN-74 }\end{array} \\
-\end{array}$} & \multirow{2}{*}{$\begin{array}{l}\text { Other } 60 \text { patients } \\
1: 64(11 / 60)\end{array}$} & \multirow{2}{*}{$\begin{array}{l}\text { Patient } \\
\text { JXHFRJ-71 } \\
-\end{array}$} & \multirow{2}{*}{\begin{tabular}{|l|}
$\begin{array}{l}\text { Other 71 } \\
\text { patients }\end{array}$ \\
$1: 64(13 / 71)$
\end{tabular}} \\
\hline \multirow{2}{*}{$\mathrm{SFGR}^{*}$} & IgG titers & & & & & \\
\hline & IgM titers & $1: 128$ & - & - & - & - \\
\hline \multirow{2}{*}{ A. phagocytophilum } & IgG titers & - & - & $1: 64(2 / 60)$ & $1: 1024$ & $1: 64(5 / 71)$ \\
\hline & IgM titers & - & - & - & $1: 80$ & - \\
\hline \multirow{2}{*}{ O. tsutsugamushi } & IgG titers & - & $1: 1024$ & $1: 64(16 / 60)$ & - & $1: 64(15 / 71)$ \\
\hline & IgM titers & - & $1: 64$ & - & - & - \\
\hline
\end{tabular}

Table 1. Serologic analysis of samples from three patients by indirect IFA, Jiangsu and Jiangxi provinces, China, 2013-2015. *SFGR: Spotted Fever Group Rickettsiae.

patients also displayed the clinical features typical of their corresponding disease (Table 2). In sum, both the PCR and IFA tests confirmed that these patients were indeed infected with the Rickettsiales bacteria recently or previously, while their recent bacterial infection was demonstrated through the appearance of the typical clinical features.

Rickettsiales bacteria identified in rodents, shrews and ticks. Rodents, shrews and ticks were concurrently collected from Yancheng and Ganzhou cities. Overall, a total of 130 mice (128 striped field mice (Apodemus agrarius) and two house mice (Mus musculus) were captured from Yancheng (Table 3). Rickettsia bacteria ( $R$. heilongjiangensis, $R$. japonica, and Uncultured Rickettsia like bacteria) and O. tsutsugamushi were identified in these rodents, with an overall prevalence of $9.23 \%$. In addition, 43 rodents including striped field mice (A. agrarius) and lesser ricefield rats (Rattus losea) and 43 Asian house shrews (Suncus murinus) were captured in Ganzhou. Rickettsia parkeri-like strain, R. raoultii, A. phagocytophilum, Ehrlichia sp. and "Candidatus Neoehrlichia mikurensis" were identified in these rodents and shrews, with an overall prevalence of $13.95 \%$.

Finally, ticks were also collected from both Yancheng and Ganzhou cites: 213 adult Haemaphysalis longicornis ticks (117 male, 96 female) were sampled from Yancheng, while 145 adult Rhipicephalus microplus ticks (65 male, 80 female) were collected in Ganzhou. Interestingly, only $R$. japonica was identified in these ticks sampled from both regions.

Phylogenetic analysis of Rickettsiales. Phylogenetic analysis of the recovered bacterial sequences revealed a diverse array of Rickettsiales bacteria in Yancheng and Ganzhou. In the rrs tree (Fig. 3A), six determinded or candidatus species of Rickettsia (R. heilongjiangensis, R. japonica, R. monacensis, Rickettsia parkeri-like strain, $R$. raoultii, and Uncultured Rickettsia like bacteria) could be defined. Notably, the sequence R. monacensis JSHMFN-77/Patient recovered from the patient in Yancheng was closely related to $R$. monacensis WHCUQA-97 previously identified in Culex quinquefasciatus mosquito from Wuhan (Hubei province, China) ${ }^{24}$. The sequences Uncultured Rickettsia like bacteria JSMMYC-52/M. musculus, Uncultured Rickettsia like bacteria JSAAYC-39/A. 


\begin{tabular}{|l|l|l|l|}
\hline \multirow{2}{*}{ Characteristic } & Patient No. & \multicolumn{2}{l|}{} \\
\cline { 2 - 4 } & $\begin{array}{l}\text { Patient } \\
\text { JXHFRJ-71 }\end{array}$ & $\begin{array}{l}\text { Patient } \\
\text { JSHFFN-74 }\end{array}$ & $\begin{array}{l}\text { Patient } \\
\text { JSHMFN-77 }\end{array}$ \\
\hline Age & 69 & 65 & 83 \\
\hline Sex & F & F & M \\
\hline Wildlife contact & Yes & Yes & Yes \\
\hline Fever & Yes & Yes & Yes \\
\hline Highest temperature,${ }^{\circ} \mathrm{C}$ & 39.0 & 38.8 & 39.0 \\
\hline Rigor & Yes & Yes & Yes \\
\hline Headache & Yes & No & Yes \\
\hline Dizziness & No & No & Yes \\
\hline Myalgia & Yes & Yes & Yes \\
\hline Rash & Yes & Yes & Yes \\
\hline Eschar & Yes $(5)$ & No & No \\
\hline Lymphadenopathy & Yes & Yes & No \\
\hline
\end{tabular}

Table 2. Epidemiological and clinical characteristics of patients with Rickettsiales infection in Jiangsu and Jiangxi provinces, China, 2013-2015.

\begin{tabular}{|c|c|c|c|c|c|c|c|c|}
\hline \multirow{2}{*}{\multicolumn{2}{|c|}{ Rickettsiales bacteria species }} & \multicolumn{3}{|l|}{ Jiangsu } & \multicolumn{4}{|l|}{ Jiangxi } \\
\hline & & \multirow{2}{*}{$\begin{array}{l}\begin{array}{l}\text { Apodemus } \\
\text { agrarius }\end{array} \\
2 / 128 \#\end{array}$} & \multirow{2}{*}{\begin{tabular}{|l|}
$\begin{array}{l}\text { Mus } \\
\text { musculus }\end{array}$ \\
$0 / 2$ \\
\end{tabular}} & \multirow{2}{*}{$\begin{array}{l}\text { Haemaphysalis } \\
\text { longicornis }\end{array}$} & \multirow{2}{*}{$\begin{array}{l}\begin{array}{l}A . \\
\text { agrarius }\end{array} \\
0 / 10\end{array}$} & \multirow{2}{*}{$\begin{array}{l}\begin{array}{l}\text { Rattus } \\
\text { losea }\end{array} \\
0 / 33 \\
\end{array}$} & \multirow{2}{*}{\begin{tabular}{|l|}
$\begin{array}{l}\text { Suncus } \\
\text { murinus }\end{array}$ \\
$0 / 43$ \\
\end{tabular}} & \multirow{2}{*}{$\begin{array}{l}\text { Rhipicephalus } \\
\text { microplus }\end{array}$} \\
\hline \multirow{5}{*}{ Rickettsia } & R. heilongjiangensis & & & & & & & \\
\hline & R.japonica & $2 / 128$ & $0 / 2$ & $39 / 213$ & $0 / 10$ & $0 / 33$ & $0 / 43$ & $1 / 145$ \\
\hline & Rickettsia like bacteria & $3 / 128$ & $1 / 2$ & $0 / 213$ & $0 / 10$ & $0 / 33$ & $0 / 43$ & $0 / 145$ \\
\hline & Rickettsia parkeri-like & $0 / 128$ & $0 / 2$ & $0 / 213$ & $0 / 10$ & $1 / 33$ & $0 / 43$ & $0 / 145$ \\
\hline & R. raoultii & $0 / 128$ & $0 / 2$ & $0 / 213$ & $0 / 10$ & $1 / 33$ & $0 / 43$ & $0 / 145$ \\
\hline Orientia & O.tsutsugamushi & $4 / 128$ & $0 / 2$ & $0 / 213$ & $0 / 10$ & $0 / 33$ & $0 / 43$ & $0 / 145$ \\
\hline \multirow{3}{*}{ Ehrlichia } & Ca. N. mikurensis ${ }^{*}$ & $0 / 128$ & $0 / 2$ & $0 / 213$ & $1 / 10$ & $3 / 33$ & $1 / 43$ & $0 / 145$ \\
\hline & E. chaffeensis & $0 / 128$ & $0 / 2$ & $0 / 213$ & $0 / 10$ & $1 / 33$ & $0 / 43$ & $0 / 145$ \\
\hline & Ehrlichia sp. & $0 / 128$ & $0 / 2$ & $0 / 213$ & $0 / 10$ & $1 / 33$ & $0 / 43$ & $0 / 145$ \\
\hline Anaplasma & A.phagocytophilum & $0 / 128$ & $0 / 2$ & $0 / 213$ & $2 / 10$ & $1 / 33$ & $0 / 43$ & $0 / 145$ \\
\hline \multicolumn{2}{|l|}{ Total } & $11 / 128$ & $1 / 2$ & $39 / 213$ & $3 / 10$ & $8 / 33$ & $1 / 43$ & $1 / 145$ \\
\hline
\end{tabular}

Table 3. Prevalence of Rickettsiales bacteria in small mammals and ticks in Jiangxi and Jiangsu provinces, China. * $C a$. N. mikurensis: Candidatus Neoehrlichia mikurensis. "PCR positive/Samples collected.

agrarius, Uncultured Rickettsia like bacteria JSAAYC-58/A. agrarius, and Uncultured Rickettsia like bacteria JSAAYC-35/A. agrarius recovered from mice in Yancheng formed a distinct lineage and were most closely related to $R$. heilongjiangensis and R. japonica. Additionally, the sequences $R$. raoultii JXRLGX-8/R. losea and Rickettsia parkeri-like strain JXRLYD-97/R. losea identified in lesser ricefield rats from Ganzhou were closely related to $R$. raoultii and $R$. parkeri, respectively. Finally, the remaining sequences, including one sampled from $R$. microplus in Ganzhou, were closely related to $R$. heilongjiangensis and $R$. japonica. Hence, these data clearly indicate the co-circulation of multiple human rickettsial pathogens in both two geographic regions. The sequences of the groEL gene were recovered from some rodents and ticks, and their position in the groEL phylogeny was consistent with that of the rrs tree (see Supplementary Fig. S1).

In the TSA56 gene phylogeny, O. tsutsugamushi sequences sampled from the striped field mice in Yancheng fell into two lineages (Fig. 3D). Sequence O. tsutsugamushi JSAAYC-57/A. agrarius was closely related to O. tsutsugamushi strain TT0705a isolated from a patient in Taiwan ${ }^{25}$, while the remaining sequences clustered together with those sampled from patients and animals in Taiwan and South East Asia ${ }^{25,26}$. Notably, both A. bovis and A. phagocytophilum were identified in Ganzhou city. More importantly, A. bovis sampled from one patient in Ganzhou was closely related to A. bovis clone China-chipmunk 25 and A. bovis clone Kh-Hc215, identified in the common chipmunk (JX092096.1) and ticks (JX092094.1), respectively. Hence, these bacteria could cause human disease. Interestingly, "Candidatus Neoehrlichia mikurensis" was identified in mice, rats and shrews, and was closely related to " $\mathrm{Ca}$. N. mikurensis $2011 \mathrm{FJ} 41 \mathrm{Nc}$ " previously identified in Niviventer confucianus from Fujian province (JQ359046.1). Finally, Ehrlichia sequences were also recovered from lesser ricefield rats and exhibited a close evolutionary relationship to E. chaffeensis in the rrs gene tree. However, the sequence Ehrlichia sp. JXRLSY$56 / R$. losea formed a distinct lineage in the rrs and groEL gene trees, suggesting that they may represent a new species of Ehrlichia in mice (Figs 3C and 4). 
A

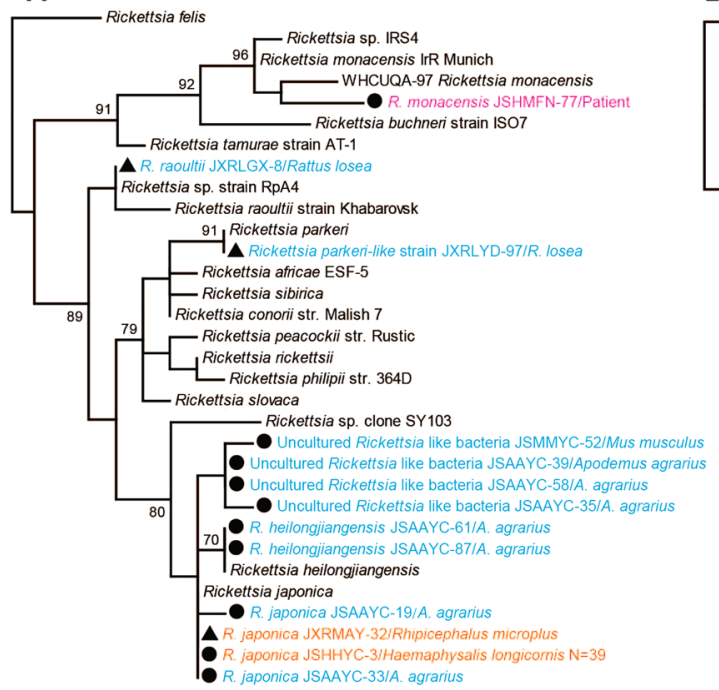

$\stackrel{\longmapsto}{\longmapsto}$

C

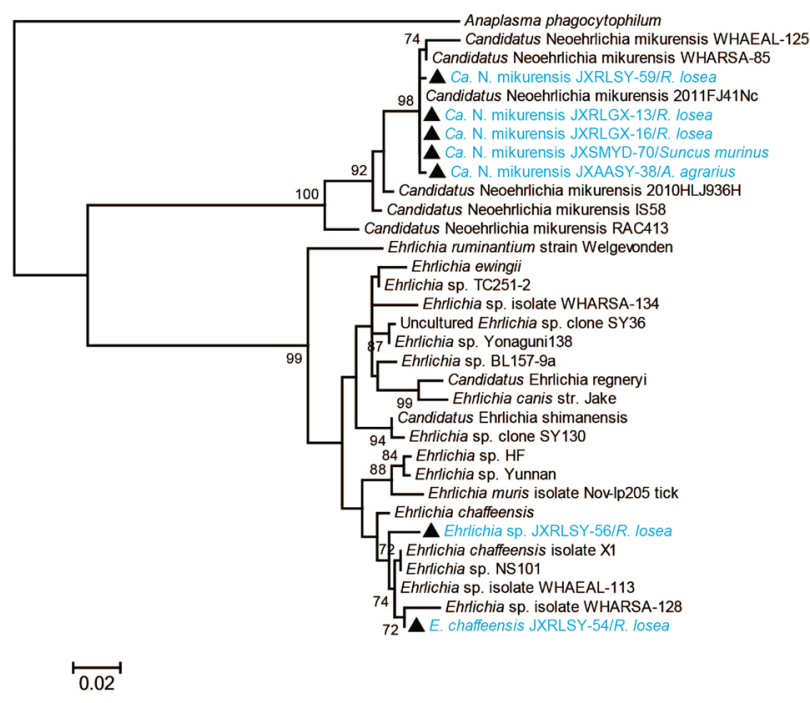

B

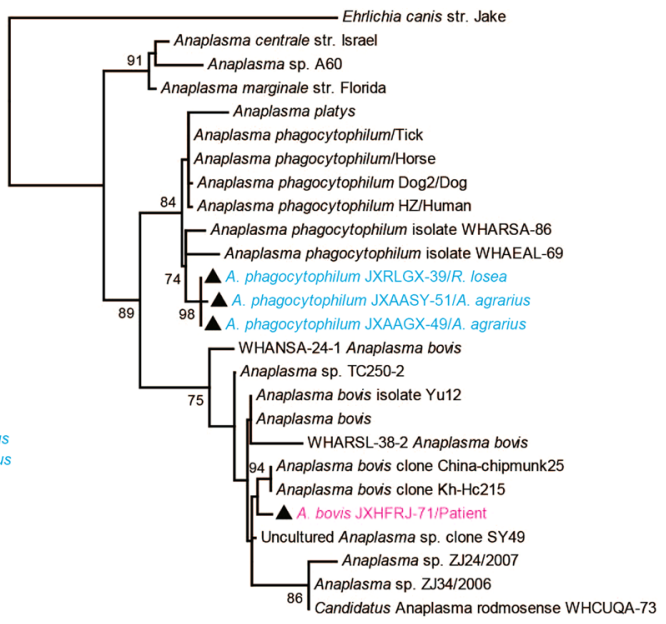

$\stackrel{\longmapsto}{0.02}$

D

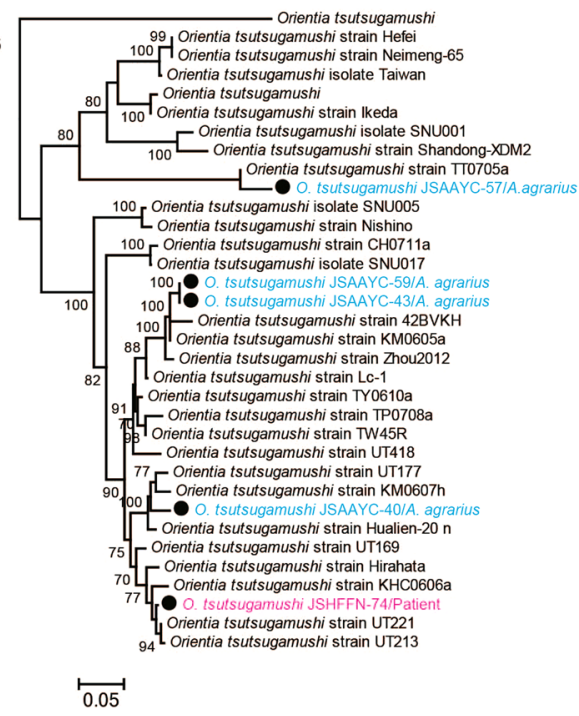

Figure 3. Phylogenetic trees based on the partial rrs gene sequences of Rickettsia (A), Anaplasma (B), Ehrlichia (C), and the TSA56 gene sequences of Orientia tsutsugamushi (D). All trees were mid-point rooted for clarity only. Bootstrap values $(>70 \%)$ are shown for appropriate nodes. The scale bar represents number of nucleotide substitutions per site. The sequences from patient samples are marked in red, those from rodents and shrews are marked in blue, and sequences from ticks are marked in orange.

\section{Discussion}

Diseases caused by Rickettsiales bacteria have caused both high morbidity and mortality in China ${ }^{27}$. Although murine typhus and epidemic typhus are considered to be controlled, at least 929 cases have been registered annually since 1999 (range 929 to 6149 cases) (unpublished data obtained from Infectious Disease Report Information Management system of China Center for Diseases Control and Prevention (CDC)). Scrub typhus initially emerged in northern China and then re-emerged in southern China ${ }^{13,16,28}$. In addition, although HGA and HME cases are commonly reported in Chinese hospitals, it is not clear how many cases have occurred each year in China as a whole. The data obtained from this study and from China CDC indicated that scrub typhus has occurred in both Yancheng and Ganzhou cities (Fig. 1), and that other diseases caused by rickettsial pathogens may be co-circulating in both localities. In addition, as we only sampled a single hospital in each region, the true number of human cases is likely to be far greater than that recorded. Thus, there is clearly an urgent need to establish a national surveillance system in China and strengthen the laboratory diagnostic in hospitals.

Currently, the genus Anaplasma contains six recognized bacterial species ${ }^{8}$. Of these, A. marginale, A. centrale and $A$. ovis are known to be ruminant-specific pathogens, while A. platys is a causative agent of infectious cyclic thrombocytopenia in dogs and cats $^{29}$. In addition, $A$. bovis is thought to be the agent of bovine ehrlichiosis, which 


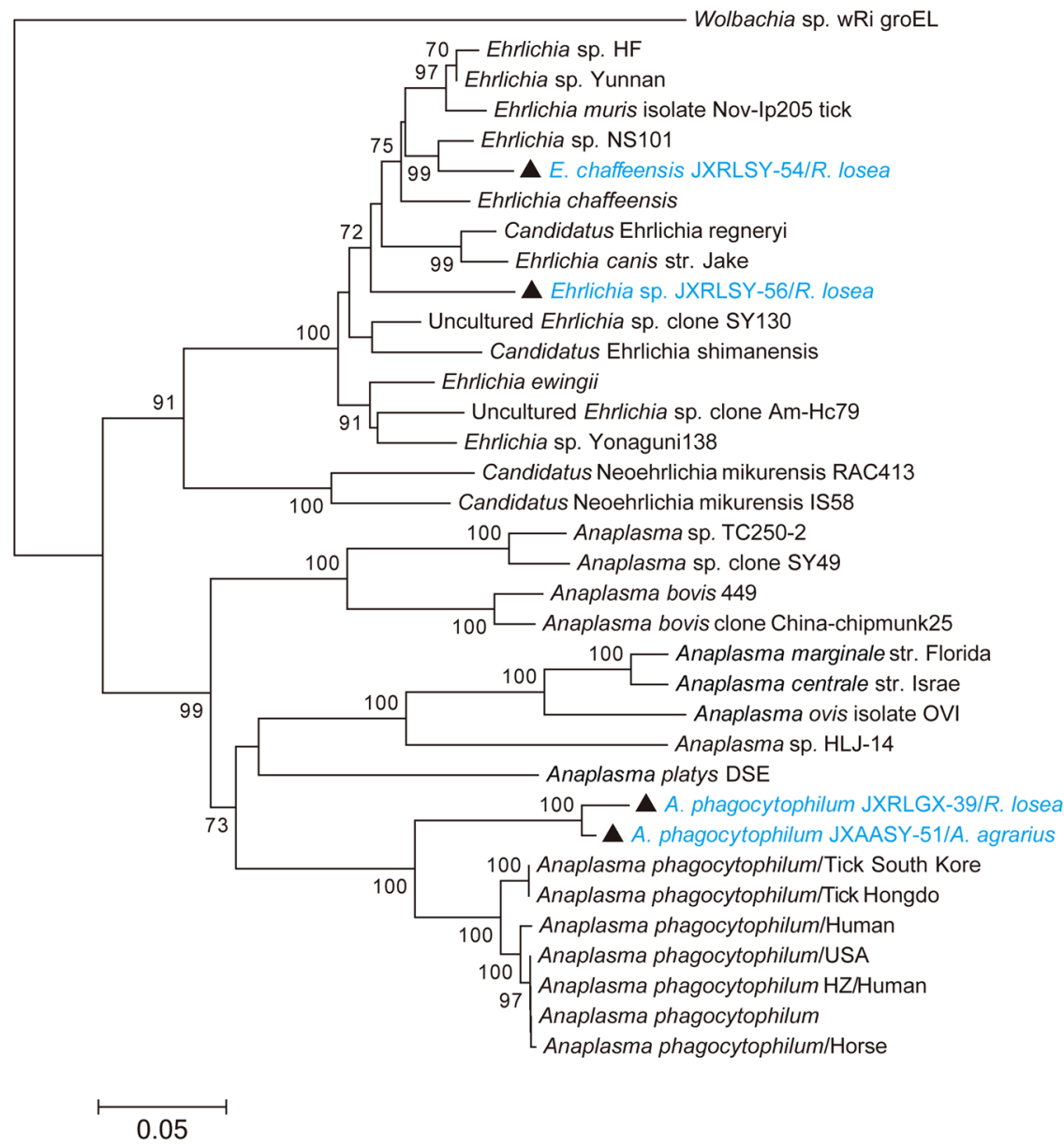

Figure 4. Phylogenetic tree based on partial groEL gene sequences of the Anaplasmataceae. The tree was midpoint rooted for clarity only. Bootstrap values $(>70 \%)$ are shown for appropriate nodes. The scale bar represents number of nucleotide substitutions per site. Taxa shown in blue were obtained from rodents and shrews.

frequently occurs in Africa and Asia ${ }^{30,31}$ and is characterized by fluctuating fever lymphadenopathy, depression, and occasionally death ${ }^{32}$. To date, only A. phagocytophilum (genus Anaplasma) is known to cause disease in humans (and sheep). Notably, however, we identified $A$. phagocytophilum in both mice and rats sampled from Ganzhou (Jiangxi province) and, remarkably, an A. bovis sequence was recovered from one patient exhibiting fever (up to $39^{\circ} \mathrm{C}$ ), rigor, headache, myalgia, rash, eschar, and lymphadenopathy. Our data therefore suggest that $A$. bovis may be the etiologic agent of human infection. Additional studies are clearly needed to determine whether this bacterium indeed causes disease in both humans and animals as in the case of A. phagocytophilum.

Spotted fever is a type of tick-borne disease caused by members of the SFGR. Several agents including $R$. heilongjiangensis, $R$. sibirica, $R$. raoultii and Candidatus $R$. tarasevichiae are known to cause this disease in China ${ }^{12,33-35}$. R. japonica harbored by multiple species of tick is also thought to cause spotted fever disease in $\operatorname{Japan}^{36}$, while R. monacensis is considered an agent of spotted fever-like disease in Europe ${ }^{1,37-39}$. Recent studies have shown that SFGR bacteria are widespread in China ${ }^{40,41}$. We identified $R$. heilongjiangensis, $R$. japonica, $R$. raoultii and Uncultured Rickettsia like bacteria in rodents and ticks from Yancheng and Ganzhou. As such, these data indicate the co-circulation of multiple members of SFGR in rodents and ticks in both two regions. More importantly, $R$. monacensis was recovered from one spotted fever patient in Yancheng, implying that the infection might be caused by this bacterium. As spotted fever-like diseases commonly observed in Chinese hospitals, especially during spring and summer, further studies are needed to determine the prevalence of SFGR bacteria in humans and animal reservoirs.

Although scrub typhus occurs only in Asia, it is estimated that more than one million cases of the disease are transmitted annually with more than one billion people at risk ${ }^{13-16,42}$. Scrub typhus has a long history in China, leading to high morbidity and mortality in humans ${ }^{27}$, particularly in southern China ${ }^{13,14,28}$. Although chiggers (family Trombiculidae) are thought to be the true reservoir of $O$. tsutsugamushi, rodents including mice and rats also play an important role in the transmission of Orientia bacteria ${ }^{43,44}$. Even though it is the only recognized species within the genus Orientia, O. tsutsugamushi exhibits high genetic diversity ${ }^{45-47}$. We identified O. tsutsugamushi in one patient as well as the striped field mice sampled from Yancheng. Notably, the recovered bacterial sequence was phylogenetically diverse (Fig. 3D), and that recovered from the patient did not cluster with those from local mice. Hence, these data suggest the co-circulation of multiple genotypes of $O$. tsutsugamushi in Yancheng. 
In conclusion, we have shown that scrub typhus is present in Yancheng city, Jiangsu province, China, while other Rickettsiales pathogens are co-circulating in both Jiangsu and Jiangxi provinces. In addition, we documented multiple species of Rickettsiales bacteria in rodents, shrews, and ticks, indicating a considerable natural diversity of Rickettsiales bacteria in both geographic regions. Hence, our data highlight the urgent need for the regular surveillance of local arthropods, mammals and humans for evidence of Rickettsiales infection in China.

\section{Materials and Methods}

Sample collection. A total of 62 blood samples were collected from patients experiencing fever at Funing People's Hospital, Yancheng city, Jiangsu province, during May 2013 to November 2015. Similarly, 72 blood samples were collected from patients presenting with fever at the First Affiliate Hospital of Gannan Medical University, Ganzhou city, Jiangxi province, China, during May 2013 to November 2015 (Fig. 2). Patients who had fever $\left(>37^{\circ} \mathrm{C}\right)$ and clinical symptoms including rash and eschar, as well as a history of wildlife contact, were enrolled and screened for infection with Rickettsiales bacteria. Patients with another obvious cause of fever (e.g., pneumonia, cellulitis, etc.), were excluded. Four $\mathrm{ml}$ whole blood with ethylenediamine tetraacetic acid dipotassium salt dihydrate (EDTA-2K) and two $\mathrm{ml}$ sera were collected from each of 134 patients. Information such as the date of the onset of illness, fever, and clinical symptoms was obtained from the relevant hospitals.

Small mammals and ticks were also collected in Yancheng and Ganzhou cities during April 2015 to October 2017. Rodents and shrews were captured with snap-traps, set at 5 meters intervals and baited with deep-fried dough sticks in both two cities. Most of ticks were directly picked from infested wild and domestic animals, although a few were collected using a tick drag-flag method. In addition, records of scrub typhus cases were obtained from the China Center for Diseases Control and Prevention.

Ethical approval. Signed individual written informed consent was obtained from each patient at the time of sample collection. All adult human subjects provided informed consent, and a parent or guardian of any child participant provided informed consent on the child's behalf (less than 18 years old). Collecting human serum samples from patients was reviewed and approved by the ethics committees of the National Institute for Communicable Disease Control and Prevention, China CDC. All methods were performed in accordance with the relevant guidelines and regulations. Animal experiments were performed according to Guidance for Experimental Animal Welfare and Ethical Treatment by the Ministry of Science and Technology of China (www. most.gov.cn/fggw/zfwj/zfwj2006/zf06yw/zf06qt/200612/t20061226_39235.htm). These protocols were approved by the National Institute for Communicable Disease Control and Prevention of the China CDC (Permit number: ICDC20170616-001). All mammals were euthanized used the ether anesthetic chamber, and all tissue samples were taken during necropsy. Tissue samples of liver, spleen, lung, and kidney were collected from small mammals for detecting Rickettsiales bacteria. Field Research was also approved by the ethics committee of National Institute of Communicable Disease Control and Prevention of the China CDC, and the field permit number is 201710082.

Serological assays. Serum samples were screened by indirect immunofluorescence assay (IFA) for IgG and IgM against spotted fever group Rickettsiae (SFGR), A. phagocytophilum, O. tsutsugamushi, Ehrlichia chaffeensis, R. prowazekii, and R. typhi by using IFA kit (Focus and Fuller, USA). As indicated in the manufacturer's instruction, IgG titers $\geq 64$ and IgM titers $\geq 20-64$ were considered as indicating a positive result.

DNA extraction and PCR assays. According to the manufacturer's instructions, $200 \mu$ l human whole blood was used to extract DNA with the QIAamp DNA Blood Mini Kit (QIAGEN, Germany). Nested or semi-nested PCRs targeting the 16S ribosomal RNA ( rrs) and $56 \mathrm{kDa}$ type-specific antigen (TSA56) genes was performed to detect Rickettsiales bacteria as described previously ${ }^{15,19}$. The $1400 \mathrm{bp}$ rrs gene was amplified using the primers Eh-out1/Eh-out2 (outer primers) and Eh-gs1/Eh-gs2 (inner primers), while the $600 \mathrm{bp}$ TSA56 gene was amplified using the primers Otr56_498F/r56_2057 (outer primers) and r56_585F/r56_2057 (inner primers). Amplified positive DNAs were purified and sequenced in both directions (Sangon, Shanghai, China).

DNA was extracted individually from all ticks using the QIAamp DNA Mini Kit (Qiagen, Germany) according to the manufacturer's instructions, and from spleen and liver tissues of small mammals using QIAamp DNeasy Blood \& Tissue Kit (Qiagen, Germany) according to the manufacturer's instructions. The spleen and liver from small mammals were homogenized. Tissue suspension $(400 \mu \mathrm{l})$ was used to extract DNA. Individual ticks were ground with $500 \mu \mathrm{l}$ or $1 \mathrm{ml}$ PBS using pestl, and the whole suspension was used to extract DNA. Rickettsiales DNA was detected using nested or semi-nested PCR targeting the rrs (length of $1400 \mathrm{bp}$ ), groEL (length of $1300 \mathrm{bp}$ ), and TSA56 (length of $900 \mathrm{bp}$ ) genes as described previously ${ }^{15,19,24}$, and fragments of the expected size were purified and sequenced in both directions (Sangon, Shanghai, China).

Sequence data and phylogenetic analyses. DNA sequences of the $r r s$, groEL, and TSA56 genes recovered from positive samples were aligned with existing reference sequences taken from GenBank using the ClustalW protocol (default parameters) as implemented in the MEGA program, version $6.06^{48}$. The bacterial sequences obtained were named according to their species, geographic origins, hosts, and sample numbers. Phylogenetic trees of the data were estimated using the Maximum Likelihood (ML) method employing the GTR $+\Gamma+$ I model of nucleotide substitution as implemented in PhyML (version 3$)^{49} .1000$ bootstrap replicates were generated to determine the level of support for individual nodes on the trees, and all trees were mid-point rooted for purposes of clarity. All sequences generated here have been submitted to GenBank and assigned accession numbers MH722222 to MH722254. 


\section{References}

1. Raoult, D. \& Parola, P. (eds) Rickettsial Diseases. (Informa Healthcare Inc. Press, 2007).

2. Parola, P. et al. Update on tick-borne rickettsioses around the world: a geographic approach. Clin. Microbiol. Rev. 26, 657-702, https://doi.org/10.1128/CMR.00032-13 (2013).

3. Chen, S. M., Dumler, J. S., Bakken, J. S. \& Walker, D. H. Identification of a granulocytotropic Ehrlichia species as the etiologic agent of human disease. J. Clin. Microbiol. 32, 589-595 (1994).

4. Maeda, M. et al. Human infection with Ehrlichia canis, a leukocytic rickettsia. N. Engl. J. Med. 16, 853-6, https://doi.org/10.1056/ NEJM198704023161406 (1987).

5. Ismail, N., Bloch, K. C. \& McBride, J. W. Human ehrlichiosis and anaplasmosis. Clin. Lab.Med. 30, 261-92, https://doi.org/10.1016/j. cll.2009.10.004 (2010).

6. Bakken, J. S. \& Dumler, J. S. Human granulocytic anaplasmosis. Infect. Dis. Clin. North. Am. 29, 341-55, https://doi.org/10.1016/j. idc.2015.02.007 (2015).

7. Li, H. et al. Human infection with a novel tick-borne Anaplasma species in China: a surveillance study. Lancet Infect. Dis. 15, 663-70, https://doi.org/10.1016/S1473-3099(15)70051-4 (2015).

8. Rar, V. \& Golovljova, I. Anaplasma, Ehrlichia, and Candidatus Neoehrlichia bacteria: pathogenicity biodiversity, and molecular genetic characteristics, a review. Infect. Genet. Evol. 11, 1842-1861, https://doi.org/10.1016/j.meegid.2011.09.019 (2011).

9. Merhej, V. \& Raoult, D. Rickettsial evolution in the light of comparative genomics. Biol. Rev. Camb. Philos. Soc. 86, 379-405, https:// doi.org/10.1111/j.1469-185X.2010.00151.x (2011).

10. Chen, X. P. et al. Detection of Wolbachia genes in a patient with non-Hodgkin's lymphoma. Clin. Microbiol. Infect. 21, 182.e1-4, https://doi.org/10.1016/j.cmi.2014.09.008 (2015).

11. Eremeeva, M. E. \& Dasch, G. A. Challenges posed by tick-borne rickettsiae: eco-epidemiology and public health implications. Front. Public. Health. 21, 55, https://doi.org/10.3389/fpubh.2015.00055 (2015).

12. Fang, L. Q. et al. Emerging tick-borne infections in mainland China: an increasing public health threat. Lancet Infect. Dis. 15, 1467-79, https://doi.org/10.1016/S1473-3099(15)00177-2 (2015).

13. Wu, Y. C. et al. Spatiotemporal Dynamics of Scrub Typhus Transmission in Mainland China, 2006-2014. PLoS. Negl. Trop. Dis. 10, e0004875, https://doi.org/10.1371/journal.pntd.0004875 (2016).

14. Sun, Y. et al. Rapid increase of scrub typhus incidence in Guangzhou, southern China, 2006-2014. BMC. Infect. Dis. 17, 13, https:// doi.org/10.1186/s12879-016-2153-3 (2017).

15. Cao, M. et al. Determination of Scrub typhus Suggests a New Epidemic Focus in the Anhui Province of China. Sci. Rep. 10, 20737, https://doi.org/10.1038/srep20737 (2016).

16. Yang, H., Bi, Z. W., Kou, Z. Q., Zheng, L. \& Zhao, Z. T. Spatial analysis of autumn-winter type scrub typhus in Shandong province, 2006-2014. Zhonghua Liu Xing Bing Xue Za Zhi. 37, 682-5, https://doi.org/10.3760/cma.j.issn.0254-6450.2016.05.019 [Article in Chinese] (2016).

17. Zhang, L. et al. Nosocomial transmission of human granulocytic anaplasmosis in China. JAMA. 300, 2263-70, https://doi. org/10.1001/jama.2008.626(2008).

18. Gao, D., Cao, W. \& Zhang, X. Investigations on Human ehrlichia infectious people in Daxingan Mountains. Zhonghua Liu Xing Bing Xue Za Zhi. 22, 137-41 [Article in Chinese] (2001).

19. Kang, Y. J. et al. Extensive diversity of Rickettsiales bacteria in two species of ticks from China and the evolution of the Rickettsiales. BMC. Evol. Biol. 30, 167, https://doi.org/10.1186/s12862-014-0167-2 (2014).

20. Ge, Y., Guo, G., Ge, B., Yin, H. \& Yin, H. The spleen microbiota of small wild mammals reveals distinct patterns with tick-borne bacteria. PLoS. Negl. Trop. Dis. 12, e0006499, https://doi.org/10.1371/journal.pntd.0006499 (2018).

21. Yang, X. J., Chen, Z. \& Liu, J. Z. The Valid Genus and Species Names of Ticks (Acari: Ixodida: Argasidae, Ixodidae) in China. Journal of Hebei Normal University (natural Science Edition). 32, 529-533 (2008). [Article in Chinese].

22. Chen, Z. et al. Ticks (acari: ixodoidea: argasidae, ixodidae) of China. Exp. Appl. Acarol. 51, 393-404, https://doi.org/10.1007/s10493010-9335-2 (2010).

23. Zhang, R. Z. et al. editors. Muridae In: Distribution of mammalian species in China, p. 185-211 (Beijing: China Forestry Publishing House, 1997).

24. Guo, W. P. et al. Extensive genetic diversity of Rickettsiales bacteria in multiple mosquito species. Sci. Rep. 9, 38770, https://doi. org/10.1038/srep38770 (2016).

25. Lu, H. Y. et al. Phylogenetic analysis of 56-kDa type-specific antigen gene of Orientia tsutsugamushi isolates in Taiwan. Am. J. Trop. Med. Hyg. 83, 658-63, https://doi.org/10.4269/ajtmh.2010.09-0608 (2010).

26. Wongprompitak, P. et al. Orientia tsutsugamushi, agent of scrub typhus, displays a single metapopulation with maintenance of ancestral haplotypes throughout continental South East Asia. Infect. Genet. Evol. 31, 1-8, https://doi.org/10.1016/j. meegid.2015.01.005 (2015).

27. Fan, M. Y., Walker, D. H., Yu, S. R. \& Liu, Q. H. Epidemiology and ecology of rickettsial diseases in the People's Republic of China. Rev. Infect. Dis. 9, 823-40 (1987).

28. Minahan, N. T., Chao, C. C. \& Tsai, K. H. The Re-Emergence and Emergence of Vector-Borne Rickettsioses in Taiwan. Trop. Med. Infect. Dis. 3, E1, https://doi.org/10.3390/tropicalmed3010001 (2017).

29. Dumler, J. S. et al. Reorganization of genera in the families Rickettsiaceae and Anaplasmataceae in the order Rickettsiales: unification of some species of Ehrlichia with Anaplasma, Cowdria with Ehrlichia and Ehrlichia with Neorickettsia, descriptions of six new species combinations and designation of Ehrlichia equi and 'HGE agent' as subjective synonyms of Ehrlichia phagocytophila. Int. J. Syst. Evol. Microbiol. 51, 2145-65, https://doi.org/10.1099/00207713-51-6-2145 (2001).

30. Harrison, A., Bastos, A. D., Medger, K. \& Bennett, N. C. Eastern rock sengis as reservoir hosts of Anaplasma bovis in South Africa. Ticks. Tick. Borne. Dis. 4, 503-5, https://doi.org/10.1016/j.ttbdis.2013.06.007 (2013).

31. Park, J. et al. Molecular detection of Anaplasma bovis in Holstein cattle in the Republic of Korea. Acta. Vet. Scand. 60, 15, https://doi. org/10.1186/s13028-018-0370-z (2018).

32. Rioche, M. Bovine rickettsiosis in Senegal. Rev. Elev. Med. Vet. Pays. Trop. 19, 485-94 (1966). [Article in French].

33. Li, H. et al. Human Infection with Novel Spotted Fever Group Rickettsia Genotype, China, 2015. Emerg. Infect. Dis. 22, 2153-2156, https://doi.org/10.3201/eid2212.160962 (2016).

34. Zhang, J. et al. First report of Rickettsia felis in China. BMC. Infect. Dis. 14, 682, https://doi.org/10.1186/s12879-014-0682-1 (2014).

35. Li, H. et al. Isolation and identification of R. raoultii in human cases: a surveillance study in three medical centers in China. Clin. Infect. Dis. 66, 1109-1115, https://doi.org/10.1093/cid/cix917 (2017).

36. Ando, S. et al. Human Rickettsia heilongjiangensis infection, Japan. Emerg. Infect. Dis. 16, 1306-8, https://doi.org/10.3201/ eid1608.100049 (2010).

37. Jado, I. et al. Rickettsia monacensis and human disease, Spain. Emerg. Infect. Dis. 13, 1405-7, https://doi.org/10.3201/eid1309.060186 (2007).

38. Madeddu, G. et al. Mediterranean spotted fever-like illness in Sardinia, Italy: a clinical and microbiological study. Infection. 44, 733-738 (2016).

39. Tijsse-Klasen, E. et al. Spotted fever group rickettsiae in Dermacentor reticulatus and Haemaphysalis punctata ticks in the UK. Parasit. Vectors. 6, 212, https://doi.org/10.1186/1756-3305-6-212 (2013). 
40. Lu, Q. B. et al. Severe Fever with Thrombocytopenia Syndrome Complicated by Co-infection with Spotted Fever Group Rickettsiae, China. Emerg. Infect. Dis. 22, 1957-1960, https://doi.org/10.3201/eid2211.161021 (2016).

41. Sun, J. et al. Detection of spotted fever group Rickettsiae in ticks from Zhejiang Province, China. Exp. Appl. Acarol. 65, 403-11, https://doi.org/10.1007/s10493-015-9880-9 (2015).

42. Luce-Fedrow, A. et al. A Review of Scrub Typhus (Orientia tsutsugamushi and Related Organisms): Then, Now, and Tomorrow. Trop. Med. Infect. Dis. 3, E8, https://doi.org/10.3390/tropicalmed3010008 (2018).

43. Bhate, R. et al. Prevalence and Phylogenetic Analysis of Orientia tsutsugamushi in Rodents and Mites from Central India. Vector. Borne. Zoonotic. Dis. 17, 749-754, https://doi.org/10.1089/vbz.2017.2159 (2017).

44. Latif, A. et al. Orientia tsutsugamushi infection in rodents in Anhui Province of China. Infect. Genet. Evol. 56, 14-18, https://doi. org/10.1016/j.meegid.2017.10.014 (2017).

45. Phetsouvanh, R. et al. Correction: The Diversity and Geographical Structure of Orientia tsutsugamushi Strains from Scrub Typhus Patients in Laos. PLoS. Negl. Trop. Dis. 10, e0004742, https://doi.org/10.1371/journal.pntd.0004742 (2016).

46. Kim, G. et al. Diversification of Orientia tsutsugamushi genotypes by intragenic recombination and their potential expansion in endemic areas. PLoS. Negl. Trop. Dis. 11, e0005408, https://doi.org/10.1371/journal.pntd.0005408 (2017).

47. Fleshman, A. et al. Comparative pan-genomic analyses of Orientia tsutsugamushi reveal an exceptional model of bacterial evolution driving genomic diversity. Microb. Genom. 4, 9, https://doi.org/10.1099/mgen.0.000199 (2018).

48. Hall, B. G. Building phylogenetic trees from molecular data with MEGA. Mol. Biol. Evol. 30, 1229-35, https://doi.org/10.1093/ molbev/mst012 (2013).

49. Guindon, S. et al. New algorithms and methods to estimate maximum-likelihood phylogenies: assessing the performance of PhyML 3.0. Syst. Biol. 59, 307-321, https://doi.org/10.1093/sysbio/syq010 (2010).

\section{Acknowledgements}

This study was supported by National Natural Science Foundation of China (grants 81672057 and 81611130073 ) and the National Science and Technology Major Project of China (No. 2018ZX10712001-006-002, 2018ZX10101002-002, and 2018ZX10305409-003-005) and E.C.H. is funded by an ARC Australian Laureate Fellowship (FL170100022).

\section{Author Contributions}

Y.Z.Z. designed the research and supervised the experiments; M.L., F.L., Y.L., J.J.S., J.M.X., Y.Z.C. and J.H.L. collected the samples and performed the experiments; M.L. and Y.Z.Z. analyzed the data; M.L., E.C.H. and Y.Z.Z. wrote the manuscript.

\section{Additional Information}

Supplementary information accompanies this paper at https://doi.org/10.1038/s41598-019-49059-3.

Competing Interests: The authors declare no competing interests.

Publisher's note: Springer Nature remains neutral with regard to jurisdictional claims in published maps and institutional affiliations.

(c) (i) Open Access This article is licensed under a Creative Commons Attribution 4.0 International License, which permits use, sharing, adaptation, distribution and reproduction in any medium or format, as long as you give appropriate credit to the original author(s) and the source, provide a link to the Creative Commons license, and indicate if changes were made. The images or other third party material in this article are included in the article's Creative Commons license, unless indicated otherwise in a credit line to the material. If material is not included in the article's Creative Commons license and your intended use is not permitted by statutory regulation or exceeds the permitted use, you will need to obtain permission directly from the copyright holder. To view a copy of this license, visit http://creativecommons.org/licenses/by/4.0/.

(C) The Author(s) 2019 